Research Article

\title{
Controllability of Impulsive Semilinear Stochastic Heat Equation with Delay
}

\author{
Hugo Leiva $\mathbb{D}^{1},{ }^{1}$ Miguel Narvaez, ${ }^{2}$ and Zoraida Sivoli $\mathbb{D}^{2,3}$ \\ ${ }^{1}$ Yachay Tech, School of Mathematical Sciences and Information Technology, Department of Mathematics, \\ San Miguel De Urcuqui 100119, Imbabura, Ecuador \\ ${ }^{2}$ Universidad De Los Andes, Facultad De Ciencias, Departamento De Matemática, Mérida 5101, Venezuela \\ ${ }^{3}$ Escuela Superior Politécnica De Chimborazo, Facultad De Ciencias, Escuela De Física Y Matematica, Riobamba, Ecuador \\ Correspondence should be addressed to Hugo Leiva; hleiva@yachaytech.edu.ec
}

Received 3 June 2020; Revised 9 November 2020; Accepted 30 November 2020; Published 17 December 2020

Academic Editor: Sining Zheng

Copyright (C) 2020 Hugo Leiva et al. This is an open access article distributed under the Creative Commons Attribution License, which permits unrestricted use, distribution, and reproduction in any medium, provided the original work is properly cited.

\begin{abstract}
LaSalle wrote the following: "it is never possible to start the system exactly in its equilibrium state, and the system is always subject to outside forces not taken into account by the differential equations. The system is disturbed and is displaced slightly from its equilibrium state. What happens? Does it remain near the equilibrium state? This is stability. Does it remain near the equilibrium state and in addition tend to return to the equilibrium? This is asymptotic stability." Continuing with what LaSalle said, we conjecture that real-life systems are always under the influence of impulses, delays, memory, nonlocal conditions, and noises, which are intrinsic phenomena no taken into account by the mathematical model that is representing by a differential equation. For many control systems in real life, delays, impulses, and noises are natural properties that do not change their behavior. So, we conjecture that, under certain conditions, the abrupt changes, delays, and noises as perturbations of a system do not modify certain properties such as controllability. In this regard, we prove the interior $S^{*}$-controllability of the semilinear stochastic heat equation with impulses and delay on the state variable, and this is done by using new techniques avoiding fixed point theorems employed by Bashirov et al.
\end{abstract}

\section{Introduction}

In this paper, we prove the interior approximate $S^{*}$-controllability of the semilinear stochastic heat equation with multiplicative noise, impulses, and delay on the state variable. This is done by using the result from Leiva [1]; Acosta
Leiva [2]; and new techniques avoiding fixed point theorems employed by Bashirov et al. [3-5]. In this regard, we will prove the interior approximate $S^{*}$-controllability of the semilinear stochastic heat equation with multiplicative noise, impulses, and delay:

$$
\left\{\begin{array}{l}
\partial_{t} z(t, x)=\left[\Delta z(t, x)+1_{\theta} u(t, x)+f(t, z(t-r, x), u(t, x))\right]+ \\
g(t, z(t-r, x), u(t, x)) \dot{m}(t, x), \quad \text { in }[0, \tau] \times \Gamma, t \neq t_{k}, \\
z(s, x)=0, \quad \text { on }(0, \tau) \times \partial \Gamma, \\
z(s, x)=\phi(s, x), \quad s \in[-r, 0], x \in \Gamma, \\
z\left(t_{k}^{+}, x\right)=z\left(t_{k}^{-}, x\right)+I_{k}\left(t_{k}, z\left(t_{k}, x\right), u\left(t_{k}, x\right)\right), \quad k=1,2,3, \ldots, p,
\end{array}\right.
$$


where $\Gamma$ is a bounded domain in $\mathbb{R}^{d}(d=1,2,3), \partial \Gamma$ denotes its boundary, $\theta$ is an open nonempty subset of $\Gamma, 1_{\theta}$ denotes the characteristic function of the set $\theta, u$ is a control processes $\mathbb{R}$-valued, the noise $\dot{m}$ is a colored noise $\mathbb{R}$ valued on $[0, \tau] \times \mathbb{R}^{d}$ with spatial correlation $q(\xi, \eta)$ given by

$$
q(\xi, \eta)=(t \wedge s)^{-1} \mathbf{E}(m(t, \xi) m(s, \eta)), \quad \xi, \eta \in \Gamma, t, s \in[0, \tau],
$$

and $\phi(\cdot, x)$ is a $\mathbb{R}$-valued $\mathscr{F}_{0}^{m}$-measurable random variable with respect to filtration $\mathscr{F}_{t}^{m}$, where $\mathscr{F}_{t}^{m}=\sigma\{m(s, x): 0 \leq s \leq \tau, x \in \Gamma\}$. The nonlinear terms $f, g, I_{k}:[0, \tau] \times \mathbb{R} \times \mathbb{R} \longrightarrow \mathbb{R}$ are smooth enough such that system (1) admits unique mild solutions for each control $u$ and satisfies the following property:

$$
\begin{gathered}
|f(t, z, u)|+|g(t, z, u)| \leq a_{0}|z|+b_{0}, \\
z \in \mathbb{R}, u \in \mathbb{R}, 0 \leq t \leq \tau, a_{0}, b_{0} \geq 0 .
\end{gathered}
$$

The term white-noise is denoted by $\dot{m}=(\partial m / \partial t)$, where $m$ is a Gaussian process $m=\left\{(t, A): t \in[0, \tau], A \in \mathscr{B}_{b}\left(\mathbb{R}^{d}\right)\right\}$ with zero mean and covariance given by (2). The noise $m$ behaves as a Brownian motion with respect to the time variable, and it has a correlated spatial covariance.

There are many practical examples of impulsive control systems which are modeled by impulsive differential equations (for more information, see the monographs: Samoilenko and Perestyuk [6]; Franco and Nieto [7]; Sun and Zhang [8]; Lakshmikanthan, Bainov and Simeonov [9]; $\mathrm{He}$ and Yu [10]; Luo and Shen [11]). The controllability of impulsive evolution equations has been studied recently for several authors, but most them study the exact controllability only (to mention, Radhakrishnan and Balachandran [12]; Chalishajar [13]; Selvi and Mallika [14]). To our knowledge, there are a few works on approximate controllability of impulsive semilinear evolution equations (to mention, Chen and Li [15] and Sakthivel and Anandhi [16]). Recently, in the study of Carrasco, Leiva, Sanchez, and Tineo [17]; Leiva [1]; Leiva and Merentes [18], the approximate controllability of semilinear evolution equations with impulses has been studied applying Rothe's fixed point theorem. Contrained controllability of finite-dimensional semilinear systems with delayed controls has been studied by Klamka $[19,20]$ where the author gives sufficient conditions for contrained local relative controllability applying a generalized open mapping theorem. Also, Klamka [21] gave necessary and sufficient conditions for different kinds of stochastic relative controllability in a given time interval which are proved for stochastic finite-dimensional linear systems with multiple delays in control.

The existence of solutions for impulsive evolution equations with delays has been studied by Hernandez, Sakthivel, and Tamaka [22]; Abada, Benchohra, and Hammouche [23]; Shikharchan and Baburao [24] and Chang $[25,26]$. Besides, impulsive and stochastic effects appear in real-life systems. Moreover, a lot of dynamical systems have structure variables subject to stochastic abrupt changes, which may result from sudden phenomena such as stochastic failures and repair of components, quick environmental changes, and changes in the interconnections of subsystems (see Mao [27]). In the stochastic context, we can mention some papers related to impulsive and delay stochastic systems: Lijuan, Junping, and Jitao [28]; Sakthivel [16]; Sukavanam and Kumar [29]; Parthasarathy and Sathya [30].

The exact and approximate controllability is known for determinist systems; but the exact controllability was introduced as a concept for linear finite-dimensional systems by Kalman in the 50s. Nevertheless, the extension of this concept to infinite dimensional systems is too strong. Therefore, the approximate controllability was introduced as a weakened version of the exact controllability. However, the exact and approximate controllability cannot be a property of stochastic systems, and this needs to be a weaker concept than the approximate controllability concepts in order to extend them to the stochastic systems. Then, the concept of the $S$-controllability is introduced. A control system is $S$-controllable, if given an arbitrary $\epsilon>0$, it is possible to steer from the point $z_{0}$ to within a distance $\sqrt{\varepsilon}$ from all points in the state space $Z$ at time $\tau$ with probability close to one. The approximate controllability and $S$-controllability concepts are equivalent for the linear system but are different for nonlinear stochastic systems. This concept and generalization are defined in Bashirov et al. [5, 31]. In this context, we used the $S^{*}$-controllability which is a weaker version of $S$-controllability.

The main objective of this article is to prove the interior $S^{*}$-controllability of the semilinear stochastic heat equation with impulses, delay, and multiplicative noises (1) simultaneously, under appropriate conditions presented above. For this, we apply the new technique presented in Bashirov et al. [3, 4, 31, 32]. In the literature, $S$-controllability for such systems, only a few works such as Bashirov and an article by Sukavanam and Kumar [29], has been reported.

\section{Preliminaries}

In this section, we introduce notations, definitions, and preliminaries which are used to write (1) as an abstract differential equation.

Let $Z, U$, and $K$ be separable Hilbert spaces and $(\Omega, \mathscr{F}, P)$ be a complete probability space with a probability measure $P$ on $\Omega$. Let $\{m(t), t \in[0, \tau]\}$ be a Wiener processes with values in $K$ and covariance nonnegative operator $Q \in L(K)(L(K)$ is the space of bounded linear operators on $K)$. If the control system is stochastic, we denote by $\mathscr{F}_{t}^{m}$ the smallest $\sigma$-field generated by $\{m(s): 0 \leq s \leq \tau\}$. We assume that there exists a complete orthonormal set $\left\{\xi_{n}, n=1,2, \ldots,\right\}$ in $K$ and a bounded sequence of nonnegative real numbers $\rho_{n}$ such that $Q \xi_{n}=\rho_{n} \xi_{n}$ with $\operatorname{Tr}(Q)=\sum_{n=1}^{\infty} \rho_{n}<\infty$. Let $\beta_{n}(t), n=1,2, \ldots$, be a sequence of real-valued one-dimensional standard Brownian motions mutually independent over $(\Omega, \mathscr{F}, P)$ such that $m(t)=\sum_{n=1}^{\infty} \sqrt{\rho_{n}} \beta_{n}(t) \xi_{n}, t \geq 0$.

Denoted by $L_{2}^{0}=L_{2}\left(Q^{(1 / 2)} K, Z\right)$, the space of all Q-Hilbert-Schmidt operators from $Q^{(1 / 2)} K$ to $Z$ with norm defined by 


$$
\|\phi\|_{L_{2}^{0}}^{2}=\operatorname{Tr}\left(\phi Q \phi^{*}\right)=\sum_{n=1}^{\infty}\left\|\sqrt{\rho_{n}} \phi \xi_{n}\right\|^{2} .
$$

$\mathbf{E}\{\cdot\}$ denotes the expectation of a random variable and $\mathbf{E}(x \mid \cdot)$ denotes the conditional expectation of $x$. Let $L_{2}\left(\Omega, \mathscr{F}_{\tau}^{m}, Z\right)$ denote the Hilbert space of all $\mathscr{F}_{\tau}^{m}$-measurable square-integrable random variables with values in $Z$. Moreover, let $L_{2}^{\mathscr{F}}([0, \tau], Z)$ denote the Hilbert space of all square-integrable and $\mathscr{F}_{t}^{m}$-measurable processes with values in $Z$ with topology given by the norm:

$$
\|z\|^{2}=\sup _{t \in[0, \tau]} \mathbf{E}\|z(t)\|_{Z}^{2}
$$

The control $u \in L_{2}^{\mathscr{F}}([0, \tau], U)$, where $L_{2}^{\mathscr{F}}([0, \tau], U)$ is the family $U$-valued measurable and $\mathscr{F}_{t}^{m}$-adapted processes with norm topology given by

$$
\|u\|^{2}=\sup _{t \in[0, \tau]} \mathbf{E}\|u(t)\|_{U}^{2} .
$$

We consider the function $z_{t}(x):[-r, 0] \longrightarrow \mathbb{R}$ defined by $z_{t}(x)(s)=z(t+s, x),-r \leq s \leq 0$ with $r>0$ being the delay. Therefore, the initial condition $\phi(s, x)$ can be written as $z_{0}=z_{0}(x)(s)=\phi(s, x), s \in[-r, 0], x \in \Gamma$.
We shall denote by $\mathbf{C}$ the set consisting of all $\mathscr{F}_{0}^{m}$-measurable bounded random processes $\phi$ with value in $Z$ : $C=\left\{\phi:[-r, 0] \longrightarrow Z, \quad \phi\right.$ bounded and $\mathscr{F}_{0}^{m}-$ measurable $\}$,

satisfying

$$
\sup _{-r \leq s \leq 0} \mathbf{E}\|\phi(s)\|_{Z}^{2}<\infty, \quad \text { with } \phi(s)(x)=\phi(s, x), x \in \Gamma .
$$

When the control system is stochastic and completely observable, then $\left\{\mathscr{F}_{t}^{m}\right\}$ is a natural filtration of $z^{u}(t)=z(t, 0, \phi, u)$. In this case, $\mathbf{E}\left(z^{u}(t) \mid \mathscr{F}_{t}^{m}\right)=z^{u}(t)$ (see Bashirov et al. [5]). So, we shall consider the following notation:

$$
S_{\tau}^{\phi}=\underset{\varepsilon>0,0 \leq p<1}{\cap} S_{\tau, \mathcal{\varepsilon}, p}^{\phi},
$$

where

$$
S_{\tau, \varepsilon, p}^{\phi}=\left\{z^{1} \in Z: \exists u \in L_{2}^{\mathscr{F}}([0, \tau], U) \text { such that } z_{0}=\phi \in C \text { and } P\left(\left\|z^{u}(\tau)-z^{1}\right\|^{2}>\varepsilon\right) \leq 1-p\right\} .
$$

Definition 1. A stochastic control system is said to be $S$-controllable if

$$
\overline{S_{\tau}^{\phi}}=\bigcap_{\varepsilon>0,0 \leq p<1} \overline{S_{\tau, \varepsilon, p}^{\phi}}=S_{\tau}^{\phi}=Z .
$$

Definition 2 (see [5]). The stochastic semilinear control system (1) is $S^{*}$-controllable if and only if, for every initial state $\phi \in \mathbf{C}, z^{1} \in Z=U=L_{2}(\Gamma)$ and $0<\sigma<\tau$, there is a sequence $u^{n}$ in $L_{2}^{\mathscr{F}}([0, \tau], U)$ such that

$$
\left\|\mathbf{E}\left[z^{u^{n}}(\tau)-z^{1} \mid \mathscr{F}_{\tau-\sigma}^{m}\right]\right\| \longrightarrow 0, \quad \text { in probability, } n \longrightarrow \infty .
$$

\section{Abstract Formulation of the Problem}

This section is devoted to set system (1) as an abstract control system in a suitable Hilbert space. To this end, we recall that the operator $A=-\Delta$ with Dirichlet boundary condition in $Z=L_{2}(\Gamma)$ has the following spectral decomposition $0<\lambda_{1}<\lambda_{2}<\cdots<\lambda_{j} \longrightarrow \infty$, where $\lambda_{j}$ denotes the eigenvalues of $A$, each one with finite multiplicity $\gamma_{j}$ equal to the dimension of the corresponding eigenspace. Therefore, the following properties for $A$ hold:

(i) For all $z \in D(A)$, we have

$$
\begin{aligned}
A z & =\sum_{j=1}^{\infty} \lambda_{j} \sum_{k=1}^{\gamma_{j}}<z, \\
\phi_{j, k}>\phi_{j, k} & =\sum_{j=1}^{\infty} \lambda_{j} E_{j} z,
\end{aligned}
$$

where $\langle\cdot, \cdot\rangle$ is the inner product in $Z,\left\{\phi_{j, k}\right\}$ is a complete orthonormal set of eigenvectors of $A$, and

$$
\begin{aligned}
& E_{n} z=\sum_{k=1}^{\gamma_{j}}<z, \\
& \phi_{j, k}>\phi_{j, k} .
\end{aligned}
$$

So, $\left\{E_{j}\right\}$ is a family of complete orthogonal projections in $Z$ and $z=\sum_{j=1}^{\infty} E_{j} z, \quad z \in Z$.

(ii) $-A$ generates an analytic semigroup $\{T(t)\}$ given by

$$
\begin{gathered}
T(t) z=\sum_{j=1}^{\infty} e^{-\lambda_{j} t} E_{j} z, \\
\|T(t)\| \leq e^{-\lambda_{1} t}, \quad t \geq 0 .
\end{gathered}
$$

Therefore, system (1) can be written as abstract functional differential equations with impulses and noses (see Acosta-Leiva [33]. 


$$
\left\{\begin{array}{l}
\mathrm{d} z(t)=\left\{-A z(t)+B_{\theta} u(t)+f^{e}\left(t, z_{t}(-r), u(t)\right)\right\} \mathrm{d} t+g^{e}\left(t, z_{t}(-r), u(t)\right) \mathrm{d} m(t), \quad z \in Z, t \geq 0 \\
z(s)=\phi(s), \quad s \in[-r, 0] \\
z\left(t_{k}^{+}\right)=z\left(t_{k}^{-}\right)+\mathscr{I}_{k}^{e}\left(t_{k}, z\left(t_{k}\right), u\left(t_{k}\right)\right), \quad k=1,2,3, \ldots, p
\end{array}\right.
$$

where $\quad u \in L_{2}^{\mathscr{F}}([0, \tau], U), \quad U=Z=K=L_{2}(\Gamma)$, $B_{v}: U \longrightarrow Z, B_{\theta} u=1_{\theta} u$ is a bounded linear operator, $z_{t} \in C([-r, 0] ; Z)$ and is defined by $z_{t}(s)=$ $z(t+s),-r \leq s \leq 0, \quad \phi \in \mathbf{C}, \quad$ and the operators $\mathscr{I}_{k}^{e}:[0, \tau] \times \quad Z \times U \longrightarrow Z$, for $k=1,2, \ldots, p$, $f^{e}:[0, \tau] \times \mathbf{C} \times U \quad \longrightarrow Z$, $g^{e}:[0, \tau] \times \mathbf{C} \times U \longrightarrow L_{2}^{0}(K, Z)$ are defined by

$$
\begin{aligned}
\mathscr{J}_{k}^{e}(t, z, u)(x) & =I_{k}(t, z(x), u(x)), \\
f^{e}(t, \phi(-r, \cdot), u(\cdot))(x) & =f(t, \phi(-r, x), u(x)), \\
g^{e}(t, \phi(-r, \cdot), u(\cdot))(x) & =g(t, \phi(-r, x), u(x)) .
\end{aligned}
$$

Proposition 1. Under condition (3) the function $f^{e}$ and $g^{e}$ satisfies

$$
\left\|f^{e}(t, \phi, u)\right\|_{Z}+\left\|g^{e}(t, \phi, u)\right\|_{L_{2}^{0}} \leq \widehat{a}_{0}\|\phi(-r)\|_{Z}+\widehat{b}_{0}, \quad 0 \leq t \leq \tau .
$$

\section{Approximate Controllability of the Linear Heat Equation}

Since the associated linear stochastic heat equation is approximately controllable in any interval of the form $[\tau-\delta, \tau]$, with $0<\delta \leq \tau$, we shall recall some properties and characterizations of the approximate controllability of linear deterministic evolution equations and linear stochastic evolution equations. In this regard, we consider the corresponding linear stochastic heat equation:

$$
\left\{\begin{array}{l}
\partial_{t} z(t, x)=\left[\Delta z(t, x)+1_{\theta} u(t, x)+\dot{m}(t, x)\right], \quad \text { in }[0, \tau] \times \Gamma, \\
z\left(t_{0}, x\right)=z_{0}(x) .
\end{array}\right.
$$

Note that, for all $z_{0}$ random variable $\mathscr{F}_{0}$-measurable and $u \in L_{2}^{\mathscr{F}}([0, \tau], U)$, the initial value problem

$$
\left\{\begin{array}{l}
\mathrm{d} z(t)=\left\{-A z(t)+B_{\theta} u(t)\right\} \mathrm{d} t+\mathrm{d} m(t), \\
z\left(t_{0}\right)=z_{0}
\end{array}\right.
$$

admits only one mild solution given by

$$
\begin{aligned}
z(t)= & z\left(t, t_{0}, z_{0}, u\right)=T(t) z_{0}+\int_{t_{0}}^{t} T(t-s) B_{\theta} u(s) \mathrm{d} s \\
& +\int_{t_{0}}^{t} T(t-s) \mathrm{d} m(s), \quad 0 \leq t_{0} \leq \tau .
\end{aligned}
$$

We also consider the deterministic system corresponding to (20), and for all $y_{0} \in Z$ and $u \in L_{2}([0, \tau], U)$, the initial value problem

$$
\left\{\begin{array}{l}
y^{\prime}=-A y(t)+B_{\theta} u(t), \quad y \in Z \\
y\left(t_{0}\right)=y_{0}
\end{array}\right.
$$

admits only one mild solution given by

$$
y(t)=y\left(t, t_{0}, y_{0}, u\right)=T(t) y_{0}+\int_{t_{0}}^{t} T(t-s) B_{\theta} u(s) \mathrm{d} s, \quad 0 \leq t_{0} \leq \tau .
$$

Definition 3. The stochastic linear system (20) is said to be approximately controllable on $[0, \tau]$ if for every initial state $z_{0} \in \mathscr{F}_{0}$ and final state $z^{1} \in L_{2}\left(\Omega, \mathscr{F}_{\tau}, Z\right)$ and any $\epsilon>0$ there exists a control $u \in L_{2}^{\mathscr{F}}([0, \tau], U), Z=U=L_{2}(\Gamma)$, such that the mild solution of (20) $z(\cdot)$ corresponding to $u$ verifies

$$
\begin{aligned}
z(0) & =z_{0}, \\
\left\|z(\tau)-z^{1}\right\|_{L_{2}\left(\Omega, \mathscr{F}_{\tau}^{m}, Z\right)} & <\mathcal{E},
\end{aligned}
$$

where

$$
\begin{aligned}
\left\|z(\tau)-z^{1}\right\|_{L_{2}\left(\Omega, \mathscr{F}_{\tau}^{m}, Z\right)} & =\mathbf{E}\left(\left\|z(\tau)-z^{1}\right\|_{Z}^{2}\right)^{(1 / 2)}, \\
\left\|z(\tau)-z^{1}\right\|_{Z}^{2} & =\int_{\Gamma}\left|z(\tau, x)-z^{1}(x)\right|^{2} \mathrm{~d} x .
\end{aligned}
$$

It is known that approximately controllability of the stochastic linear system (20) and deterministic linear system (22) for linear infinite dimensional systems are equivalent (see Mahmudov [34]). Now, we define the following operator.

Definition 4 (see [33]). For system (22), we define the following concept: the controllability maps $G_{\tau \delta}: L_{2}([\tau-\delta, \tau], U) \longrightarrow Z, G_{\delta}: L_{2}([0, \delta], U) \longrightarrow Z$ defined by

$$
\begin{aligned}
G_{\tau \delta} u & =\int_{\tau-\delta}^{\tau} T(\tau-s) B_{\theta} u(s) \mathrm{d} s, \quad u \in L_{2}([\tau-\delta, \tau], U), \\
G_{\delta} v & =\int_{0}^{\delta} T(s) B_{\theta} v(s) \mathrm{d} s, \quad v \in L_{2}([0, \delta], U),
\end{aligned}
$$

satisfy the following relation: 


$$
\begin{aligned}
G_{\tau \delta} u & =\int_{\tau-\delta}^{\tau} T(\tau-s) B_{\theta} u(s) \mathrm{d} s=\int_{0}^{\delta} T(s) B_{\theta} u(\tau-s) \mathrm{d} s \\
& =G_{\delta} u(\tau-\cdot) .
\end{aligned}
$$

The adjoint of these operators $G_{\tau \delta}^{*}: Z \longrightarrow L_{2}([\tau-\delta, \tau], U), G_{\delta}^{*}: Z \longrightarrow L_{2}([0, \delta], U)$ are given by

$$
\begin{aligned}
\left(G_{\tau \delta}^{*} z\right)(t) & =B_{\theta}^{*} T^{*}(\tau-t), \quad t \in[\tau-\delta, \tau], \\
\left(G_{\delta}^{*} z\right)(t) & =B_{\theta}^{*} T^{*}(t), \quad t \in[0, \delta] .
\end{aligned}
$$

The controllability operators $Q_{\delta}: Z \longrightarrow Z$ are given by

$$
Q_{\delta}(z)=G_{\delta} G_{\delta}^{*}(z)=\int_{0}^{\delta} T(t) B_{\theta} B_{\theta}^{*} T^{*}(t) \mathrm{d} t .
$$

$Q_{\tau \delta}: Z \longrightarrow Z$ is defined by

$$
Q_{\tau \delta}(z)=G_{\tau \delta} G_{\tau \delta}^{*}(z)=\int_{\tau-\delta}^{\tau} T(\tau-t) B_{\theta} B_{\theta}^{*} T^{*}(\tau-t) \mathrm{d} t=Q_{\delta}(z) .
$$

The following lemma holds in general for a linearbounded operator $G: W \longrightarrow Z$ between Hilbert spaces $W$ and $Z$ (see Bashirov et al. [5]; Curtain and Pritchard [35]; Curtain and Zwart [36] and Leiva et al. [37]).

Lemma 1 The following statements are equivalent to the approximate controllability of the linear system (20) on $[\tau-\delta, \tau]$.
(a) $\overline{\text { Range }\left(G_{\tau \delta}\right)}=Z$
(b) $\operatorname{Ker}\left(G_{\tau \delta}^{*}\right)=\{0\}$
(c) $\left\langle Q_{\tau \delta} z, z\right\rangle>0, z \neq 0$ in $Z$
(d) $\lim _{\alpha \longrightarrow 0^{+}} \alpha\left(\alpha I+Q_{\tau \delta}\right)^{-1} z=0$

Remark 1. Lemma 1 implies that, for all $z \in Z$, we have

$$
G_{\tau \delta} u_{\alpha}=z-\alpha\left(\alpha I+Q_{\tau \delta}\right)^{-1} z,
$$

where $u_{\alpha}=G_{\tau \delta}^{*}\left(\alpha I+Q_{\tau \delta}\right)^{-1} z, \alpha \in(0,1]$.

So, $\lim _{\alpha \longrightarrow 0} G_{\tau \delta} u_{\alpha}=z$ and the error $E_{\tau \delta} z$ of this approximation is given by the formula:

$$
E_{\tau \delta} z=\alpha\left(\alpha I+Q_{\tau \delta}\right)^{-1} z, \quad \alpha \in(0,1],
$$

and the family of linear operators $\Gamma_{\alpha \tau \delta}: Z \longrightarrow W$, defined for $0<\alpha \leq 1$ by

$$
\Gamma_{\alpha \tau \delta} z=G_{\tau \delta}^{*}\left(\alpha I+Q_{\tau \delta}\right)^{-1} z,
$$

is an approximate inverse for the right of the operator $G_{\tau \delta}$, in the sense that

$$
\lim _{\alpha \longrightarrow 0} G_{\tau \delta} \Gamma_{\alpha \tau \delta}=I,
$$

in the strong topology.

Lemma 2. $Q_{\tau \delta}>0$ if and only if linear system (22) is approximately controllable on $[\tau-\delta, \tau]$. Moreover, given an initial state $y_{0} \in Z$ and a final state $Z^{1}$, we can find a sequence of controls $\left\{u_{\alpha}\right\}_{0<\alpha \leq 1} \subset L_{2}(\tau-\delta, \tau ; U)$, where

$$
u_{\alpha}=G_{\tau \delta}^{*}\left(\alpha I+Q_{\tau \delta}\right)^{-1}\left(y^{1}-T(\tau) y_{0}\right), \quad \alpha \in(0,1],
$$

such that the solutions $y(t)=y\left(t, \tau-\delta, y_{0}, u_{\alpha}\right)$ of the initial value problem

$$
\left\{\begin{array}{l}
y^{\prime}=\left\{A y+B u_{\alpha}(t)\right\}, \quad y \in Z, t>0 \\
y(\tau-\delta)=y_{0}
\end{array}\right.
$$

satisfies

$$
\lim _{\alpha \longrightarrow 0^{+}} y\left(\tau, \tau-\delta, y_{0}, u_{\alpha}\right)=z^{1},
$$

i.e.,

$$
\lim _{\alpha \longrightarrow 0^{+}} y(\tau)=\lim _{\alpha \longrightarrow 0^{+}}\left\{T(\delta) y_{0}+\int_{\tau-\delta}^{\tau} T(\tau-s) B u_{\alpha}(s) \mathrm{d} s\right\}=z^{1} .
$$

\section{5. $S^{*}$-Controllability of the Semilinear Stochastic System}

In this section, we shall prove the main result of this paper, the interior $S^{*}$-controllability of the heat equation with impulses, delay, and multiplicative noise (1), which is equivalent to prove the $S^{*}$-controllability of system (16). To this end, for all $\phi \in \mathbf{C}$ and $u \in L_{2}^{\mathscr{F}}([0, \tau], U)$, the initial value problem

$$
\left\{\begin{array}{l}
\mathrm{d} z=\left\{-A z+B_{\theta} u+f^{e}\left(t, z_{t}(-r), u(s)\right)\right\} \mathrm{d} t+g^{e}\left(t, z_{t}(-r), u(t)\right) \mathrm{d} m(t), \quad z \in Z, 0 \leq t \leq \tau, \\
z(s)=\phi(s), \quad s \in[-r, 0], \\
z\left(t_{k}^{+}\right)=z\left(t_{k}^{-}\right)+\mathcal{F}_{k}^{e}\left(t_{k}, z\left(t_{k}\right), u\left(t_{k}\right)\right), \quad k=1,2,3, \ldots, p,
\end{array}\right.
$$


admits only one mild solution given by

$$
\begin{aligned}
z^{u}(t)= & T(t) \phi(0)+\int_{0}^{t} T(t-s) B_{\theta} u(s) \mathrm{d} s+\int_{0}^{t} T(t-s) f^{e}(s, z(s-r), u(s)) \mathrm{d} s \\
& +\int_{0}^{t} T(t-s) g^{e}(s, z(s-r), u(s)) \mathrm{d} m(s)+\sum_{0<t_{k}<t} T\left(t-t_{k}\right) \mathscr{F}_{k}^{e}\left(t_{k}, z\left(t_{k}\right), u\left(t_{k}\right)\right),
\end{aligned}
$$

with $0 \leq t \leq \tau$.

$$
\mathbf{P}\left(\left\|\mathbf{E}\left(z^{u^{n}}(\tau) \mid \mathscr{F}_{\tau-\sigma}^{m}\right)-z^{1}\right\|_{Z}>\varepsilon\right) \longrightarrow 0, \quad \text { as } n \longrightarrow \infty .
$$

Proposition 2 (see [37]). If $\overline{\text { Range }(G)}=Z$, then

$$
\sup _{\alpha>0}\left\|\alpha\left(\alpha I+G_{\tau \delta} G_{\tau \delta}^{*}\right)^{-1}\right\| \leq 1 \text {. }
$$

Now, we are ready to present and prove the main result of this paper, the $S^{*}$-controllability of the semilinear heat equation with impulses, delay, and multiplicative noise.

Theorem 1. Under condition (3), the semilinear heat equations with impulses, delays, and multiplicative noise (1) is $S^{*}$-controllable on $[0, \tau]$.

Proof. Given $\phi \in \mathbf{C}$, a final state $z^{1} \in Z$, and $\epsilon>0$, we want to find a sequence of control $\left\{u^{n}\right\} \subset L_{2}^{\mathscr{F}}([0, \tau], U)$ steering the system from $\phi(0)$ to an $\epsilon$-neighborhood of $z^{1} \in Z$ on time $\tau$ in probability. Precisely, for $0<\delta<\min \left\{\tau-t_{p}, r\right\}=\sigma<\tau$, there exists control $\left\{u^{n}\right\} \in L_{2}^{\mathscr{F}}([0, \tau], U)$ such that the corresponding solution of (39) satisfies
Consider any process control $u \in L_{2}^{\mathscr{F}}([0, \tau], U)$ and the corresponding solution $z^{\delta, \alpha}(t)=z\left(t, 0, \phi, u_{\alpha}^{\delta}\right)$ of the initial value problem (39). For $\alpha \in(0,1]$, we define the control $u_{\alpha}^{\delta}$ as

$$
u_{\alpha}^{\delta}(t]= \begin{cases}u(t), & \text { if } 0 \leq t \leq \tau-\delta \\ u_{\alpha}(t), & \text { if } \tau-\delta<t \leq \tau\end{cases}
$$

where

$$
u_{\alpha}(t)=B_{\theta}^{*} T^{*}(\tau-t)\left(\alpha I+G_{\tau \delta} G_{\tau \delta}^{*}\right)^{-1}\left(z^{1}-T(\delta) z(\tau-\delta)\right),
$$

$u_{\alpha}^{\delta} \in L_{2}^{\mathscr{F}}([0, \tau], U)$ since $z(\tau-\delta)$ is $\mathscr{F}_{\tau-\delta^{-}}$measurable and $z^{1} \in Z$.

Now, since $0<\delta<\tau-t_{p}$, the corresponding solution $z^{\delta, \alpha}(t)=z\left(t, 0, \phi, u_{\alpha}^{\delta}\right)$ of the initial value problem (39) at time $\tau$ can be written as follows:

$$
\begin{aligned}
z^{\delta, \alpha}(\tau)= & T(\tau) \phi(0)+\int_{0}^{\tau} T(\tau-s) B_{\theta} u_{\alpha}^{\delta}(s) \mathrm{d} s+\int_{0}^{\tau} T(\tau-s) f^{e}\left(s, z^{\delta, \alpha}(s-r), u_{\alpha}^{\delta}(s)\right) \mathrm{d} s \\
& +\int_{0}^{\tau} T(t-s) g^{e}\left(s, z^{\delta, \alpha}(s-r), u_{\alpha}^{\delta}(s)\right) \mathrm{d} m(s)+\sum_{0<t_{k}<\tau} T\left(\tau-t_{k}\right) \mathscr{J}_{k}^{e}\left(t_{k}, z\left(t_{k}\right), u_{\alpha}^{\delta}\left(t_{k}\right)\right) .
\end{aligned}
$$

Therefore,

$$
\begin{aligned}
z^{\delta, \alpha}(\tau)= & T(\delta)\left\{T(\tau-\delta) \phi(0)+\int_{0}^{\tau-\delta} T(\tau-\delta-s) B_{\theta} u_{\alpha}^{\delta}(s) \mathrm{d} s\right. \\
& +\int_{0}^{\tau-\delta} T(\tau-\delta-s) f^{e}\left(s, z^{\delta, \alpha}(s-r), u_{\alpha}^{\delta}\right) \mathrm{d} s \\
& +\int_{0}^{\tau-\delta} T(t-\delta-s) g^{e}\left(s, z^{\delta, \alpha}(s-r), u_{\alpha}^{\delta}\right) \mathrm{d} m(s) \\
& \left.+\sum_{0<t_{k}<\tau-\delta} T\left(\tau-\delta-t_{k}\right) \mathscr{J}_{k}^{e}\left(z^{\delta, \alpha}\left(t_{k}\right), u_{\alpha}^{\delta}\left(t_{k}\right)\right)\right\}+\int_{\tau-\delta}^{\tau} T(\tau-s) B_{\theta} u_{\alpha}^{\delta}(s) \mathrm{d} s \\
& +\int_{\tau-\delta}^{\tau} T(\tau-s) f^{e}\left(s, z^{\delta, \alpha}(s-r), u_{\alpha}^{\delta}(s)\right) \mathrm{d} s \\
& +\int_{\tau-\delta}^{t} T(t-s) g^{e}\left(s, z^{\delta, \alpha}(s-r), u_{\alpha}^{\delta}(s)\right) \mathrm{d} m(s) .
\end{aligned}
$$


Hence,

$$
\begin{aligned}
z^{\delta, \alpha}(\tau)= & T(\delta) z(\tau-\delta)+\int_{\tau-\delta}^{\tau} T(\tau-s) B_{\theta} u_{\alpha}(s) \mathrm{d} s+\int_{\tau-\delta}^{\tau} T(\tau-s) f^{e}\left(s, z^{\delta, \alpha}(s-r), u_{\alpha}(s)\right) \mathrm{d} s \\
& +\int_{\tau-\delta}^{t} T(t-s) g^{e}\left(s, z^{\delta, \alpha}(s-r), u_{\alpha}(s)\right) \mathrm{d} m(s) .
\end{aligned}
$$

Thus,

$$
\begin{aligned}
z^{\delta, \alpha}(\tau)= & T(\delta) z(\tau-\delta)+\int_{\tau-\delta}^{\tau} T(\tau-s) B_{\theta} u_{\alpha}(s) \mathrm{d} s+\int_{\tau-\delta}^{\tau} T(\tau-s) f^{e}\left(s, z^{\delta, \alpha}(s), u_{\alpha}(s)\right) \mathrm{d} s \\
& +\int_{\tau-\delta}^{t} T(t-s) g^{e}\left(s, z^{\delta, \alpha}(s-r), u_{\alpha}(s)\right) \mathrm{d} m(s) .
\end{aligned}
$$

Also, the corresponding mild solution $y^{\delta, \alpha}(t)=y(t, \tau-$ $\left.\delta, z(\tau-\delta), u_{\alpha}\right)$ of the initial value problem of linear solution (22) at time $\tau$ is given by

$$
y^{\delta, \alpha}(\tau)=T(\delta) z(\tau-\delta)+\int_{\tau-\delta}^{\tau} T(\tau-s) B_{\theta} u_{\alpha}(s) \mathrm{d} s,
$$

or equivalently

$$
y^{\delta, \alpha}(\tau)=T(\delta) z(\tau-\delta)+\int_{\tau-\delta}^{\tau} T(\tau-s) B_{\theta} B_{\theta}^{*} T^{*}(\tau-s)\left(\alpha I+G_{\tau \delta} G_{\tau \delta}^{*}\right)^{-1}\left(z^{1}-T(\delta) z(\tau-\delta)\right) \mathrm{d} s .
$$

Since $z(\tau-\delta)$ is $\mathscr{F}_{\tau-\delta}^{m}$-measurable, and also $y^{\delta, \alpha}(\tau)$ is $\mathscr{F}_{\tau-\delta}^{m}$-measurable, it is measurable with respect to the smaller $\sigma$ field $\mathscr{F}_{\tau-\sigma}^{m}$. On the other hand, we have that

$$
y^{\delta, \alpha}(\tau)-z^{1}=\alpha\left(\alpha I+G_{\tau \delta} G_{\tau \delta}^{*}\right)^{-1}\left(T(\delta) z(\tau-\delta)-z^{1}\right),
$$

Taking conditional expectation with respect to $\mathscr{F}_{\tau-\sigma}^{m}$ and using the fact that the term

$$
\int_{\tau-\delta}^{\tau} T(t-s) g^{e}\left(s, z^{\delta, \alpha}(s-r), u_{\alpha}^{\delta}(s)\right) \mathrm{d} m(s),
$$

is independent of $\mathscr{F}_{\tau-\sigma}^{m}$, we have

and subtracting (49) from (48), we get

$$
\begin{aligned}
z^{\delta, \alpha}(\tau)-y^{\delta, \alpha}(\tau)= & \int_{\tau-\delta}^{\tau} T(\tau-s) f^{e}\left(s, z^{\delta, \alpha}(s-r), u_{\alpha}^{\delta}(s)\right) \mathrm{d} s \\
& +\int_{\tau-\delta}^{\tau} T(t-s) g^{e}\left(s, z^{\delta, \alpha}(s-r), u_{\alpha}^{\delta}(s)\right) \mathrm{d} m(s) .
\end{aligned}
$$

$$
\mathbf{E}\left(z^{\delta, \alpha}(\tau)-y^{\delta, \alpha}(\tau) \mid \mathscr{F}_{\tau-\sigma}^{m}\right)=\mathbf{E}\left(\int_{\tau-\delta}^{\tau} T(\tau-s) f^{e}\left(s, z^{\delta, \alpha}(s-r), u_{\alpha}^{\delta}(s)\right) \mathrm{d} s \mid \mathscr{F}_{\tau-\sigma}^{m}\right) .
$$


Therefore, putting $M=\sup _{0 \leq t \leq \tau}\|T(t)\|$ and applying Jensen' inequality, we have that

$$
\begin{aligned}
\left\|\mathbf{E}\left[z^{\delta, \alpha}(\tau)-y^{\delta, \alpha}(\tau) \mid \mathscr{F}_{\tau-\sigma}^{m}\right]\right\|_{Z}^{2} & =\left\|\mathbf{E}\left[\int_{\tau-\delta}^{\tau} T(\tau-s) f^{e}\left(s, z^{\delta, \alpha}(s-r), u_{\alpha}^{\delta}(s)\right) \mathrm{d} s \mid \mathscr{F}_{\tau-\sigma}^{m}\right]\right\|_{Z}^{2} \\
& \leq\left(\mathbf{E}\left\|\int_{\tau-\delta}^{\tau} T(\tau-s) f^{e}\left(s, z^{\delta, \alpha}(s-r), u_{\alpha}^{\delta}(s)\right) \mathrm{d} s \mid \mathscr{F}_{\tau-\sigma}^{m}\right\|\right)^{2} \\
& \leq\left(\mathbf{E}\left[\int_{\tau-\delta}^{\tau}\|T(\tau-s)\|_{Z}\left\|f^{e}\left(s, z^{\delta, \alpha}(s-r), u_{\alpha}^{\delta}(s)\right)\right\|_{Z} \mathrm{~d} s \mid \mathscr{F}_{\tau-\sigma}^{m}\right]\right)^{2} \\
& \leq M^{2}\left(\int_{\tau-\delta}^{\tau}\left\{\tilde{a}_{0}\left\|z^{\delta, \alpha}(s-r)\right\|_{Z}+\tilde{b}_{0}\right\} \mathrm{d} s\right)^{2} \\
& =M^{2}\left(\tilde{a}_{0} \int_{\tau-\delta}^{\tau}\left\|z^{\delta, \alpha}(s-r)\right\|_{Z} \mathrm{~d} s+\int_{\tau-\delta}^{\tau} \tilde{b}_{0} \mathrm{~d} s\right)^{2} .
\end{aligned}
$$

If we take $0<\delta<r$ and $\tau-\delta<s<\tau$, then $s-r<\tau-r<\tau-\delta$ and $z^{\delta, \alpha}(s-r)=z(s-r)$. So, we obtain the following estimate:

$$
\left\|\mathbf{E}\left[z^{\delta, \alpha}(\tau)-y^{\delta, \alpha}(\tau) \mid \mathscr{F}_{\tau-\sigma}^{m}\right]\right\|_{Z}^{2} \leq M^{2}\left(\widetilde{a}_{0} K_{1} \delta+\widetilde{b}_{0} \delta\right)^{2} \leq 2 M^{2} \delta^{2}\left(\tilde{a}_{0}^{2} K_{1}^{2}+\widetilde{b}_{0}^{2}\right)
$$

where $K_{1}=\sup _{0 \leq s \leq \tau}\|z(s-r)\|_{Z}$.

Hence,

$$
\begin{aligned}
\left\|\mathbf{E}\left[z^{\delta, \alpha}(\tau)-z^{1} \mid \mathscr{F}_{\tau-\sigma}^{m}\right]\right\|_{Z}^{2} & =\left\|\mathbf{E}\left[z^{\delta, \alpha}(\tau)-y^{\delta, \alpha}(\tau) \mid \mathscr{F}_{\tau-\sigma}^{m}\right]+\mathbf{E}\left[y^{\delta, \alpha}(\tau)-z^{1} \mid \mathscr{F}_{\tau-\sigma}^{m}\right]\right\|_{Z}^{2} \\
& \leq\left(\left\|\mathbf{E}\left[z^{\delta \cdot \alpha}(\tau)-y^{\delta, \alpha}(\tau) \mid \mathscr{F}_{\tau-\sigma}^{m}\right]\right\|_{Z}+\left\|\mathbf{E}\left[y^{\delta \cdot \alpha}(\tau)-z^{1} \mid \mathscr{F}_{\tau-\sigma}^{m}\right]\right\|_{Z}\right)^{2} \\
& \leq 2\left\|\mathbf{E}\left[z^{\delta \cdot \alpha}(\tau)-y^{\delta, \alpha}(\tau)\left|\mathscr{F}_{\tau-\sigma}^{m}\right|\right]\right\|_{Z}^{2}+2\left\|\mathbf{E}\left[y^{\delta, \alpha}(\tau)-z^{1} \mid \mathscr{F}_{\tau-\sigma}^{m}\right]\right\|_{Z}^{2} \\
& \leq 4 M^{2} \delta^{2}\left(\tilde{a}_{0}^{2} K_{1}^{2}+\widetilde{b}_{0}^{2}\right)+2\left\|\mathbf{E}\left[y^{\delta \cdot \alpha}(\tau)-z^{1} \mid \mathscr{F}_{\tau-\sigma}^{m}\right]\right\|_{Z}^{2}
\end{aligned}
$$

or equivalently, by orthogonal projection property of conditional expectation, we have that

$$
\mathbf{E}\left\|\mathbf{E}\left[z^{\delta \cdot \alpha}(\tau)-z^{1} \mid \mathscr{F}_{\tau-\sigma}^{m}\right]\right\|_{Z}^{2} \leq 4 M^{2} \delta^{2}\left(\tilde{a}_{0}^{2} K_{1}^{2}+\widetilde{b}_{0}^{2}\right)+2 \mathbf{E}\left\|y^{\delta, \alpha}(\tau)-z^{1}\right\|_{Z}^{2}
$$

From equation (51), Lemma 1(d), and Proposition 2, we get that

$$
\begin{aligned}
\left\|y^{\delta, \alpha}(\tau)-z^{1}\right\|_{Z}^{2} & =\left\|\alpha\left(\alpha I+G_{\tau \delta} G_{\tau \delta}^{*}\right)^{-1}\left(T(\delta) z^{\delta, \alpha}(\tau-\delta)-z^{1}\right)\right\|_{Z}^{2} \\
& \leq\left\|\alpha\left(\alpha I+G_{\tau \delta} G_{\tau \delta}^{*}\right)^{-1}\right\|_{Z}^{2}\left\|\left(T(\delta) z^{\delta, \alpha}(\tau-\delta)-z^{1}\right)\right\|_{Z}^{2} \\
& \leq\left\|T(\delta) z^{\delta, \alpha}(\tau-\delta)\right\|_{Z}^{2}+\left\|z^{1}\right\|_{Z}^{2} .
\end{aligned}
$$


Then, $\left\|y^{\delta, \alpha}(\tau)-z^{1}\right\|$ is dominated by an integrable random variable. Consequently, for every $0<\delta<\tau$,

$$
\mathrm{E}\left\|y^{\delta, \alpha}(\tau)-z^{1}\right\|_{Z}^{2} \longrightarrow 0, \quad \text { as } \alpha \longrightarrow 0^{+}, \delta \longrightarrow 0^{+} \text {. }
$$

Therefore, for fixed $0<\sigma<\tau$, we can choose $0<\delta_{n}<\sigma$ such that

$$
M^{2} \delta_{n}^{2}\left(\tilde{a}_{0}^{2} K_{1}^{2}+\tilde{b}_{0}^{2}\right) \leq \frac{1}{8 n}
$$

There exists $\alpha_{n}>0$ such that

$$
\mathbf{E}\left\|y^{\delta_{n}, \alpha_{n}}(\tau)-z^{1}\right\|_{Z}^{2}<\frac{1}{4 n} .
$$

Then, there exists a sequence of controls $u_{\alpha_{n}}^{\delta_{n}} \in L_{2}^{\mathscr{F}}([0, \tau], U)$ such that

$$
\left\|\mathbf{E}\left[z^{\delta_{n}, \alpha_{n}}(\tau)-z^{1} \mid \mathscr{F}_{\tau-\sigma}^{m}\right]\right\|^{2}=\mathbf{E}\left\|\mathbf{E}\left[z^{\delta_{n}, \alpha_{n}}(\tau)-z^{1} \mid \mathscr{F}_{\tau-\sigma}^{m}\right]\right\|_{Z}^{2} \leq \frac{1}{n} \longrightarrow 0, \quad n \longrightarrow \infty .
$$

$$
|f(t, z, u)|+|g(t, z, u)| \leq \rho(z), \quad u, z \in \mathbb{R}
$$

Since mean square convergence implies convergence in probability, we obtain

$\left\|\mathbf{E}\left[z^{\delta_{n}, \alpha_{n}}(\tau)-z^{1} \mid \mathscr{F}_{\tau-\sigma}^{m}\right]\right\| \longrightarrow 0, \quad$ in probability, $n \longrightarrow \infty$.

This completes the proof of the theorem.

\section{Conclusions}

In this article the approximate $S^{*}$-controllability was proved for the stochastic semilinear heat equation with impulse, delay, and multiplicative noise. For this, we avoid the method of fixed point theorems by applying a new alternative method due to Bashirov et al. This technique can be used to prove the $S^{*}$-controllability of the stochastic Benjamin Bona Mohany equation with impulses and delays, for the stochastic strongly damped wave equation under influence of impulses and delays and stochastic partial differential equations modelling the structural damped vibrations of a string or beam under the influence of impulses and delays.

Considering Theorem 1 , there are many systems that do not satisfy the sufficient condition (3) and still controllable. In fact, if we change condition (3) by the following condition:

$$
|f(t, z, u)|+|g(t, z, u)| \leq a|z|^{\alpha}+b|u|^{\beta}+c, \quad u, z \in \mathbb{R}
$$

where

$$
\begin{aligned}
& \frac{1}{2} \leq \alpha<1, \\
& \frac{1}{2} \leq \beta<1,
\end{aligned}
$$

the system is still $S^{*}$-controllable by using ideas from Leiva et al. [1, 2, 37].

Moreover, if we observe carefully the proof of Theorem 1 , it can be concluded that condition (3) could be replaced by the following more general one: where $\rho: \mathbb{R} \longrightarrow[0, \infty)$ is a continuous function. In particular, $\rho(z)=|z|^{2}$.

\section{Data Availability}

The data used in the research to support the findings of this study are purely Bibliographic and from scientific publications, which are included in the article with their respective citations.

\section{Conflicts of Interest}

The authors declare that they have no conflicts of interest.

\section{References}

[1] H. Leiva, "Approximate controllability of semilinear impulsive evolution equations," Abstract and Applied Analysis, vol. 2015, Article ID 797439, 7 pages, 2015.

[2] H. Leiva, "Controllability of the semilinear heat equatin with impulses and delay on the state," Nonautonomous Dynamical Systems, vol. 2, pp. 52-62, 2015.

[3] A. E. Bashirov and N. Ghahramanlou, "On partial complete controllability of semilinear systems," Abstract and Applied Analysis, vol. 2013, Article ID 52105, 8 pages, 2013.

[4] A. E. Bashirov and N. Ghahramanlou, "On partial approximate controllability of semilinear systems," Cogent Engineering, vol. 1, no. 1, 2014.

[5] A. E. Bashirov, N. Mahmudov, N. Semi, and H. Etikan, "On partial controllability concepts," Inernational Journal of Control, vol. 80, no. 1, pp. 1-7, 2007.

[6] A. M. Samoilenko and N. A. Perestyuk, "Impulsive differential equations," World Scientific Series on Nonlinear Science Series A, vol. 14, 1995.

[7] D. Franco and J. J. Nieto, "Maximum principles for periodic impulsive first order problems," Journal of Computational and Applied Mathematics, vol. 88, no. 1, pp. 149-159, 1998.

[8] J. Sun and Y. Zhang, "Impulsive control of a nuclear spin generator," Journal of Computational and Applied Mathematics, vol. 157, no. 1, pp. 235-242, 2003.

[9] V. Lakshmikantham, D. D. Bainov, and P. S. Simeonov, Theory of Impulsive Differential Equations, World Scientific, Singapore, Asia, 1989.

[10] Z. He and J. Yu, "Periodic boundary value problem for firstorder impulsive functional differential equations," Journal of 
Computational and Applied Mathematics, vol. 138, no. 2, pp. 205-217, 2002.

[11] Z. Luo and J. Shen, "Stability results for impulsive functional differential equations with infinite delays," Journal of Computational and Applied Mathematics, vol. 131, no. 1-2, pp. 55-64, 2001.

[12] B. Radhakrishnan and K. Balachandran, "Controllability results for semilinear impulsive integro differential evolution systems with nonlocal conditions," Journal of Control Theory and Applications, vol. 10, no. 1, pp. 28-34, 2012.

[13] D. N. Chalishajar, "Controllability of impulsive partial neutral funcional differential equation with infinite delay," International Journal of Mathematical Analysis, vol. 5, no. 5, pp. 369-380, 2011.

[14] S. Selvi and M. M. Arjunan, "Controllability results for impulsive differential systems with finite delay," Journal of Nonlinear Sciences and Applications, vol. 5, no. 3, pp. 206-219, 2012.

[15] L. Chen and G. Li, "Approximate controllability of impulsive differential equations with nonlocal conditions," International Journal of Nonlinear Science, vol. 10, no. 4, pp. 438-446, 2010.

[16] R. Sakthivel, "Controllability of nonlinear impulsive Ito type stochastic systems," International Journal of Applied Mathematics and Computer Science, vol. 19, no. 4, pp. 589-595, 2009.

[17] A. Carrasco, H. Leiva, J. L. Sanchez, and M. A. Tineo, "Approximate controllability of the semilinear impulsive beam equation with impulses," Transaction on IoT and Cloud Computing, vol. 2, no. 3, pp. 70-88, 2014.

[18] H. Leiva and N. Merentes, "Approximate controllability of the impulsive semilinear heat equation," Journal of Mathematics and Applications, vol. 38, pp. 85-104, 2015.

[19] J. Klamka, "Contrained controllability of the semilinear systems with delay in control," Bulletin of the Polish Academy of Sciences: Technical Sciences, vol. 56, no. 4, pp. 333-337, 2008.

[20] J. Klamka, "Stochastic controllability and minimum energy control of systems with multiple delays in control," Applied Mathematics and Computation, vol. 206, no. 2, pp. 704-715, 2008.

[21] J. Klamka, "Contrained controllability of the semilinear systems with delayed controls," Nonlinear Dynamics, vol. 56, no. 1-2, pp. 169-177, 2009.

[22] E. Hernandez, R. Sakthivel, and S. M. Tanaka Aki, "Existence results for impulsive evolution differential equations with state-dependent delay," Electronic Journal of Differential Equations, vol. 2008, no. 28, p. 11, 2008.

[23] N. Abada, M. Benchohra, and H. Hammouche, "Existence results for semilinear differential evolution equations with impulses and delay," CUBO A Mathematical Journal, vol. 12, no. 2, pp. 1-17, 2010 .

[24] R. Jain and M. B. Dhakne, "On mild solutions of nonlocal semilinear impulsive functional integro-differential equations," Applied Mathematics E-Notes, vol. 13, pp. 109-119, 2014.

[25] Y.-K. Chang, "Controllability of impulsive functional differential systems with infinite delay in Banach spaces," Chaos, Solitons \& Fractals, vol. 33, no. 5, pp. 1601-1609, 2007.

[26] Y.-K. Chang, A. Anguraj, and M. Mallika Arjunan, "Controllability of impulsive neutral functional differential inclusions with infinite delay in Banach spaces," Chaos, Solitons \& Fractals, vol. 39, no. 4, pp. 1864-1876, 2009.

[27] X. Mao, Stochastic Differential Equations and Applications, Elis Horwood, Chichester, England, 1997.
[28] L. Shen, J. Shi, and J. Sun, "Complete controlability of impulsive stochastic integro-differential systems," Automatica, vol. 46, no. 6, pp. 1068-1073, 2010.

[29] N. Sukavanam and M. Kumar, "S-controllability of an abstract first order semilinear control system," Numerical Functional Analysis and Optimization, vol. 31, no. 9, pp. 1023-1034, 2010.

[30] K. Balachandran and R. Sathya, "Controllability of nonlocal impulsive stochastic quasi-linear integro differential systems," Electronic Journal of Qualitative Theory of Differential Equations, vol. 2011, no. 50, pp. 1-16, 2011.

[31] A. E. Bashirov and K. R. Kerimov, "On controllability conception for stochastic systems," SIAM Journal on Control and Optimization, vol. 35, no. 2, pp. 384-398, 1997.

[32] A. E. Bashirov and N. Ghahramanlou, "On partial Scontrollability of semilinear partially observable systems," Inernational Journal of Control, vol. 88, no. 5, pp. 969-982, 2014.

[33] A. Acosta and H. Leiva, "Robustness of the controllability for the heat equation under the influence of multiple impulses and delays," Quaestiones Mathematicae, vol. 41, no. 6, pp. 761-772, 2018.

[34] N. I. Mahmudov, "Controllability of linear stochastic systems in Hilbert spaces," Journal of Mathematical Analysis and Applications, vol. 259, no. 1, pp. 64-82, 2001.

[35] R. F. Curtain and A. J. Pritchard, "Infinite dimensional linear systems," in Lecture Notes in Control and Information Sciences, Vol. 8, Springer-Verlag, Berlin, Germany, 1978.

[36] R. F. Curtain and H. J. Zwart, "An introduction to infinite dimensional linear systems theory," in Text in Applied Mathematics, Vol. 21, Springer-Verlag, New York, NY, USA, 1995.

[37] H. Leiva, N. Merentes, and J. Sanchez, "Characterization of semilinear dense range operators and applications," Abstract and Applied Analysis, vol. 2013, Article ID 729093, 11 pages, 2013. 